\title{
Diffusion-Driven Device for a High-Resolution Dose-Response Profiling of Combination Chemotherapy
}

\author{
Alexander Ganser, ${ }^{t, \neq}$ Günter Roth,$^{\perp, \otimes}$ Joost C. van Galen, ${ }^{\S}$ Janneke Hilderink, ${ }^{\dagger}$ \\ Joost J. G. Wammes, ${ }^{\dagger}$ Ingo Müller," Frank N. van Leeuwen, ${ }^{\mathbb{\$}}$ Karl-Heinz Wiesmüller, ${ }^{\circ}$ and \\ Roland Brock*,t, $\perp$
}

\begin{abstract}
Department of Biochemistry, Nijmegen Centre for Molecular Life Sciences, Radboud University Nijmegen Medical Centre, Nijmegen, The Netherlands, Department of Pediatrics, Laboratory of Pediatric Oncology, Radboud University Nijmegen Medical Centre, Nijmegen, The Netherlands, Department of General Pediatrics, Hematology and Oncology, University Children's Hospital, Tübingen, Germany, Department of Molecular Biology, Interfaculty Institute for Cell Biology, Eberhard Karls University Tübingen, Tübingen, Germany, and EMC Microcollections GmbH, Tübingen, Germany
\end{abstract}

Combination therapies have proven vital in the fight against HIV and cancer. However, the identification and optimization of such combination therapies is largely experience driven and an activity of clinicians rather than of systematic screening efforts. Here we present a diffusion device, compatible with the format of a 12-well microtiter plate, to create and test all possible mixtures of two substances with only two pipetting steps. Applications to the testing of different drug combinations and the parallel screening of different leukemia cell lines as well as primary patient cells are presented. The diffusion device yields qualitatively and quantitatively comparable results to an MTT viability assay conducted in a standard 96-well format albeit with a tremendous reduction of processing steps. In addition, a fluorescence-based annexin $\mathrm{V}$ binding assay of cell death was implemented. Next to the reduction of processing steps, the diffusion device constitutes a considerable assay miniaturization that overcomes the problems typically associated with miniaturization as a consequence of small sample volumes. Given its ease of handling, the device will greatly advance the development and optimization of combination drugs and the identification of optimum drug combinations in personalized medicine.

Over the past years, the use of drug combinations rather than

* To whom correspondence should be addressed. Department of Biochemistry, Nijmegen Centre for Molecular Life Sciences, Radboud University Nijmegen Medical Centre, P.O. Box 9101, 6500 HB Nijmegen, The Netherlands. E-mail: r.brock@ncmls.ru.nl.

${ }^{\dagger}$ Department of Biochemistry, Nijmegen Centre for Molecular Life Sciences, Radboud University Nijmegen Medical Centre.

† Present address: EMC Microcollections GmbH, Tübingen, Germany.

$\S$ Department of Pediatrics, Laboratory of Pediatric Oncology, Radboud University Nijmegen Medical Centre.

"University Children's Hospital, Tübingen.

${ }^{\perp}$ Department of Molecular Biology, Interfaculty Institute for Cell Biology, Eberhard Karls University Tübingen.

${ }^{\otimes}$ Present address: Department of Microsystems Engineering, Institute of Microsystem Technology (IMTEK), University of Freiburg, Freiburg.

EMC microcollections $\mathrm{GmbH}$. single agents in the treatment of various diseases, including cancer, has been increasing. ${ }^{1}$ On one hand, only multitargeted therapies effectively meet the challenges associated with drug resistance. On the other hand, because of the high costs involved in developing novel drugs, combinations of therapeutics with wellestablished safety records provide a short-cut to therapeutics with sometimes new and unexpected activities. ${ }^{2}$

To this point, the testing of combinations in drug discovery has been based on standard pipetting and assay techniques. ${ }^{2}$ However, such standard techniques are severely limited when it comes to the acquisition of high-resolution dose-response profiles. For mixtures of two compounds, the number of pipetting steps scales quadratically with the number of concentrations tested, precluding the acquisition of detailed dose-response profiles for a larger number of different compound combinations. Moreover, reagent consumption also scales quadratically, making assay miniaturization highly desirable. Finally, there is a strong motivation in personalized medicine to acquire information on dose-response profiles of drugs. ${ }^{3}$

The formation of concentration gradients using diffusion and microfluidic systems represented a first step toward the microreactor-based generation of substance mixtures. ${ }^{4-8}$ However, these systems do not enable the generation of all possible mixtures of two compounds, a prerequisite for a detailed understanding of drug interactions. Here, we present a diffusion-based device to

(1) Zimmermann, G. R.; Lehar, J.; Keith, C. T. Drug Discovery Today 2007, $12,34-42$.

(2) Borisy, A. A.; Elliott, P. J.; Hurst, N. W.; Lee, M. S.; Lehár, J.; Price, E. R.; Serbedzija, G.; Zimmermann, G. R.; Foley, M. A.; Stockwell, B. R.; Keith, C. T. Proc. Natl. Acad. Sci. U.S.A. 2003, 100, 7977-7982.

(3) Trusheim, M. R.; Berndt, E. R.; Douglas, F. L. Nat. Rev. Drug Discovery 2007, 6, 287-293.

(4) Chung, B. G.; Lin, F.; Jeon, N. L. Lab Chip 2006, 6, 764-768.

(5) Fujii, S.; Uematsu, M.; Yabuki, S.; Abo, M.; Yoshimura, E.; Sato, K. Anal. Sci. 2006, 22, 87-90.

(6) Keenan, T. M.; Folch, A. Lab Chip 2008, 8, 34-57.

(7) Mosadegh, B.; Huang, C.; Park, J. W.; Shin, H. S.; Chung, B. G.; Hwang, S. K.; Lee, K. H.; Kim, H. J.; Brody, J.; Jeon, N. L. Langmuir 2007, 23, 10910-10912.

(8) Rosoff, W. J.; McAllister, R.; Esrick, M. A.; Goodhill, G. J.; Urbach, J. S. Biotechnol. Bioeng. 2005, 91, 754-759. 


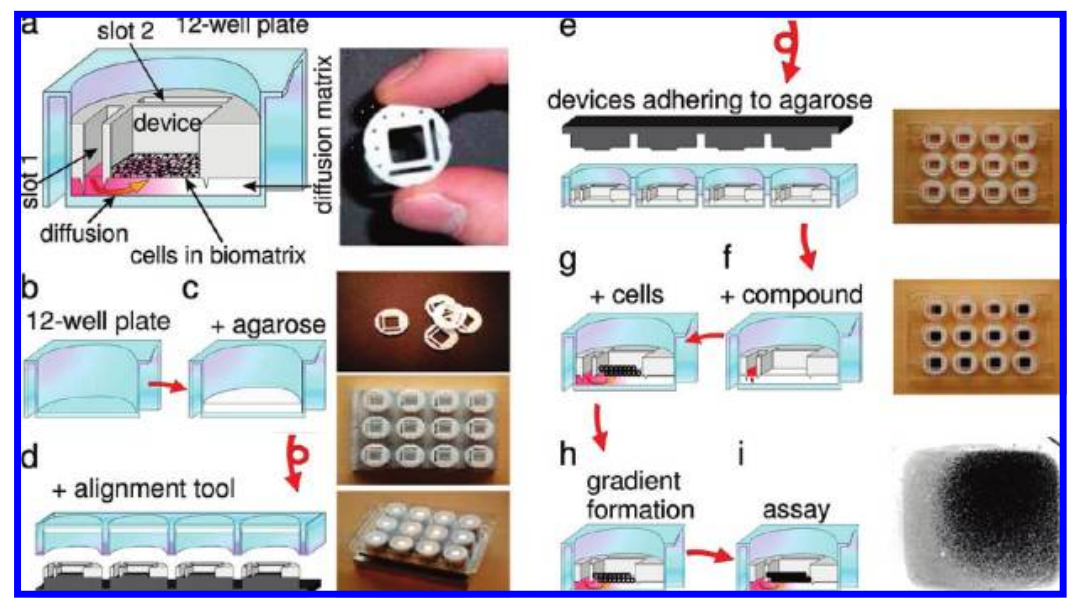

Figure 1. Design and operating principle of the diffusion device. (a) The diffusion device enables the formation and testing of orthogonally superimposed concentration gradients. Test substances are filled into application slots that lie next to the central assay area. Anchor burrs anchor the device in the diffusion matrix, typically a layer of agarose or gelatin, to ensure that concentration gradients form by convection-free diffusion only. $(\mathrm{b}-\mathrm{i})$ Operating principle for the formation and cellular testing of 2D gradients. (b,c) First, a polymeric diffusion matrix, such as agarose is poured into the wells of a 12-well plate. (d) After solidification of the agarose, the diffusion devices, placed on an alignment tool, are pressed onto the agarose and $(e)$ the device is turned around. $(f, g)$ Then the compound solutions are filled into the application slots and the gradient $(\mathrm{h})$ is either preformed before addition of cells or both, substances and cells are added at the same time. For assays such as the detection of cellular viability using an MTT-test, cells are embedded into a collagen hydrogel to preserve the spatial information of the viability in dependence of the drug gradient. The converted MTT-substrate can be imaged by a low-magnifying stereomicroscope (i). Right panels: Photographs of the individual steps of the procedure.

create and analyze all possible mixtures of two substances in a defined concentration range with only two pipetting steps.

For the application of this device in the identification of optimum combinations of drugs and drug candidates, several issues had to be addressed: Typically, biologically active substances are tested over a concentration range of $2-3$ orders of magnitude. Moreover, in standard, microtiter plate-based assays each concentration of a test compound is contained within a separate well. For use in the diffusion device, assays had to be adapted to the continuous format of the diffusion matrix. Finally, gradients generated by diffusion are dynamic over time. Therefore, it was required to confirm that nevertheless drug activities determined with the device conform to activities determined with a standard microtiter plate-based assay.

By testing different combinations of chemotherapeutics, we here demonstrate that the diffusion device fulfills all these requirements. An MTT cell viability test ${ }^{9}$ was readily adapted to the format of the diffusion device, yielding results that were in qualitative and quantitative agreement with the results obtained by a standard assay. Furthermore, the feasibility of an annexin V-based assay of cell death is demonstrated. Our experiments demonstrate that even though it may be counterintuitive, the concentration gradients are sufficiently stable for conducting a complex cellular assay. In its simplicity and ease of handling, the diffusion device therefore represents a significant advancement for the recording of complex dose-response functions to substance mixtures using a minimum of cells and substances.

\section{EXPERIMENTAL SECTION}

Preparation of the Diffusion Device. The diffusion devices were manufactured by computer-aided design (CAD) from polyoxymethylene (trade name Delrin, DuPont, Bad Homburg, Germany) by the workshop of the Institute of Applied Physics,

(9) Mosmann, T. L. Immunol. Methods 1983, 65, 55-63.
University of Tübingen. Before use in the diffusion assay, the devices were sterilized with $70 \%(\mathrm{v} / \mathrm{v})$ ethanol and air-dried in a laminar flow tissue culture hood. Then, for placement into a 12well plate, the devices were arranged on an alignment tool (Figure 1d). Agarose (low melting point agarose, Sigma Aldrich Chemie, Taufkirchen, Germany) was suspended in RPMI 1640 medium (Pan Biotech, Aidenbach, Germany) to a concentration of 1.5\% (w/v). After boiling, $550 \mu \mathrm{L}$ of molten agarose were poured into each well of a 12-well plate (Greiner Bio-One, Frickenhausen, Germany). After 6-8 min, when the agarose was sufficiently solid, the 12-well plate was pressed upside down onto the alignment tool (Figure 1d). The alignment tool enabled control of the penetration depth of the devices into the agarose and the parallel alignment of all devices to each other. The assembly was flipped over, and the alignment tool was lifted off (Figure 1e). Stock solutions of rhodamine B and fluorescein sodium salt (Fluka, Buchs, Switzerland) were prepared in PBS (pH 7.0) or water. The chemotherapeutics were diluted in RPMI 1640 before use.

Fluorescence Microscopy. Fluorophore gradients were imaged by epifluorescence microscopy using an inverted fluorescence microscope (Axiovert 100 M, Carl Zeiss, Jena) equipped with a mercury arc lamp for excitation of fluorescence, a SensiCam cooled 12-bit CCD camera (PCO Computer Optics, Kelheim, Germany), and a Plan Neofluar 10× NA 0.3 lens. For detection of fluorescein, a filter set consisting of an HQ470/40 excitation filter, a Q495LP dichroic mirror, and an HQ525/50 emission filter was used. Rhodamine $B$ was detected with a filter set consisting of an HQ548/10 excitation filter, a Q565LP dichroic mirror, and an HQ610/75 emission filter (all filters from Chroma, Fürstenfeldbruck, Germany). Gradients were imaged by recording 10 frames along the direction of the gradient. To validate the uniformity of the gradients, three such image series were recorded in parallel using a self-programmed macro implemented in Metamorph (Universal Imaging, Downingtown, PA). 
For recording of doxorubicin gradients, fluorescence was excited with the $488 \mathrm{~nm}$ line of an argon ion laser and detected from 500 to $600 \mathrm{~nm}$ using a confocal microscope (TCP SP5, Leica, Mannheim). Tile scans of the assay area were recorded at a focusing depth of $200 \mu \mathrm{m}$ with a 10× NA 0.3 HCX PL Fluotar. A calibration curve for doxorubicin intensities was acquired for doxorubicin concentrations of $1 \mu \mathrm{M}$ to $1 \mathrm{mM}$ in medium containing 10\% FCS (Pan Biotech) in a 96-well plate. The fluorescence intensity profiles in the diffusion device were converted into concentrations based on the calibration curve.

Phosphatidylserine exposure was detected using annexin V-AlexaFluor 647 (Invitrogen, Karlsruhe, Germany). Prior to the embedding in the collagen, cells were stained by incubation with $1 \mu \mathrm{M}$ carboxyfluorescein-succinimidylester diacetate (CFSE-DA, Invitrogen) according to the protocol provided by the manufacturer. A volume of $3 \mu \mathrm{L}$ of the annexin $\mathrm{V}$ stock solution was diluted into the collagen matrix. Microscopy was performed using an LSM510 confocal laser scanning microscope (Carl Zeiss) equipped with a PlanNeofluar $10 \times 0.3 \mathrm{NA}$ and a closed pinhole in order to reduce blurr caused by out-of-focus light. Single cells were imaged with an LD $40 \times 0.6$ NA lens.

Cell Culture and Image Generation. All cells were cultured with RPMI 1640 medium containing $10 \% \mathrm{FCS}$. A total of $7.5 \times 10^{5}$ cells per device were resuspended in $75 \mu \mathrm{L}$ of gel medium (2 parts of FCS and HEPES (0.2 mM, Sigma) and 1 part of 10fold RPMI 1640) and mixed with $25 \mu \mathrm{L}$ of collagen type 1 from rat tail (kindly provided by Heike Mertsching, Fraunhofer Institut for Interfacial Engineering and Biotechnology, Stuttgart, Germany). Cryopreserved primary leukemic cells from a child diagnosed with T-lineage acute lymphoblastic leukemia (TALL), obtained after informed consent, were thawed and allowed to recover for at least $1 \mathrm{~h}$ prior to embedding in collagen. After the addition of collagen, the $100 \mu \mathrm{L}$ cell suspension was pipetted immediately into the devices and the devices were placed into the tissue culture incubator. Within $5 \mathrm{~min}$, the collagen formed a hydrogel on which a further 200 $\mu \mathrm{L}$ of medium were pipetted. The drug solution was pipetted into the application slots at the same time. The application slots accommodated $10-50 \mu \mathrm{L}$ of drug solution. In all experiments described here, $20 \mu \mathrm{L}$ were used. After $16 \mathrm{~h}$ of incubation, the medium was replaced with $1 \mathrm{mg} / \mathrm{mL}$ MTT-solution in PBS (Sigma) and incubated for another $2 \mathrm{~h}$. Finally, the MTTsolution was removed, the devices were lifted off from the agarose matrix, and the collagen hydrogels with the cells were covered with a cover-glass. The covered gels were imaged with a stereomicroscope (MZ FL III, Leica) with 1-fold magnification in gray scale mode with a DC 200 camera (Leica). The images were processed with the image analysis software ImagePro Plus (Media Cybernetics, Bethesda, MD).

Analysis of Concentration Gradients. On the basis of calibration samples of known concentration, fluorescence profiles of fluorescein, rhodamine $\mathrm{B}$, and doxorubicin were converted into absolute concentrations (Supporting Information). Plots of concentration versus distance from the substance reservoir were fitted with Origin 6.11 (OriginLab, Northampton) with a complementary error function (eq 1),

$$
y=c_{0}\left(1-\operatorname{erf}\left(\frac{\left(x-x_{0}\right)}{2 \sqrt{D t}}\right)\right)+y_{0}
$$

where $y$ is the concentration at position $x$ (measured from the edge of the application slot) $c_{0}$ is the concentration in the diffusion matrix underneath the reservoir, erf is the error function, $x_{0}$ is the position of the reservoir, $y_{0}$ accounts for an offset caused by autofluorescence of the gel, $t$ corresponds to the duration of diffusion, and $D$ is the diffusion constant. The values $x, y$, and $t$ were known. All other values were obtained from the fit. (Figure S-1 in the Supporting Information).

\section{RESULTS}

Design of the Diffusion Device. So far, the acquisition of high-resolution dose-response profiles for mixtures of two biologically active compounds requires processing steps that scale quadratically with the number of combinations to be tested. As a consequence, such tests heavily depend on high-performance pipettors, precluding the acquisition of dose-response profiles for a larger number of different substance combinations and in clinical settings. Therefore, it was our goal to develop a robust device to provide such information with a minimum of processing steps. In order to be useful as a tool in screening applications and cellular diagnostics, a rugged design that is accessible to cheap manufacturing as a disposable is required. Following this design philosophy, we developed a "diffusion device" (Figure 1). The design of the device was inspired by orthogonally oriented linear gradients used in the semiconductor industry to create photonic wave guides, codiffusion transistors, and flashable memory cells. ${ }^{10,11}$ In our case, the first devices were manufactured by computer-aided design from the inert plastic polyoxymethylene. In the meantime, the device has become available as a consumable produced from polycarbonate by injection molding.

The device is placed on a flat gel that acts as a diffusion matrix. Test substances are pipetted into application slots adjacent to a central assay area, in which the activity of the substance mixtures is tested. Diffusion from a reservoir represents a well-understood physical process for the generation of a substance gradient. Because of the perpendicular orientation of the slots, in the central assay area, a superposition of the two concentration gradients is formed that comprises all possible combinations of the two substances. The device fits into 1 well of a 12-well plate (Figure 1). Proper alignment of 12 devices and uniform penetration depth into the diffusion matrix are ensured by an alignment tool, enabling the reliable testing of a large number of different substance pairs.

Validation of the Diffusion Device for the Generation of Substance Gradients. In order to describe concentration gradients in the diffusion device over time, two conditions had to be met: First, it had to be validated, that for drugs and druglike molecules, gradient formation could be well described by diffusion laws. Second, we needed to determine how the concentration of a substance in the application slot related to the concentration in the diffusion matrix. With this information at hand, it should be

(10) Reisch, M.; Itoh, K.; Lee, T.; Sakurai, T.; Sansen, W. M. C.; SchmittLandsiedel, D. High-Frequency Bipolar Transistors: Physics, Modeling, Applications; Springer: Berlin, Germany, 2003.

(11) Widmann, D.; Mader, H.; Friedrich, H. Technology of Integrated Circuits; Springer: Berlin, Germany, 2000. 


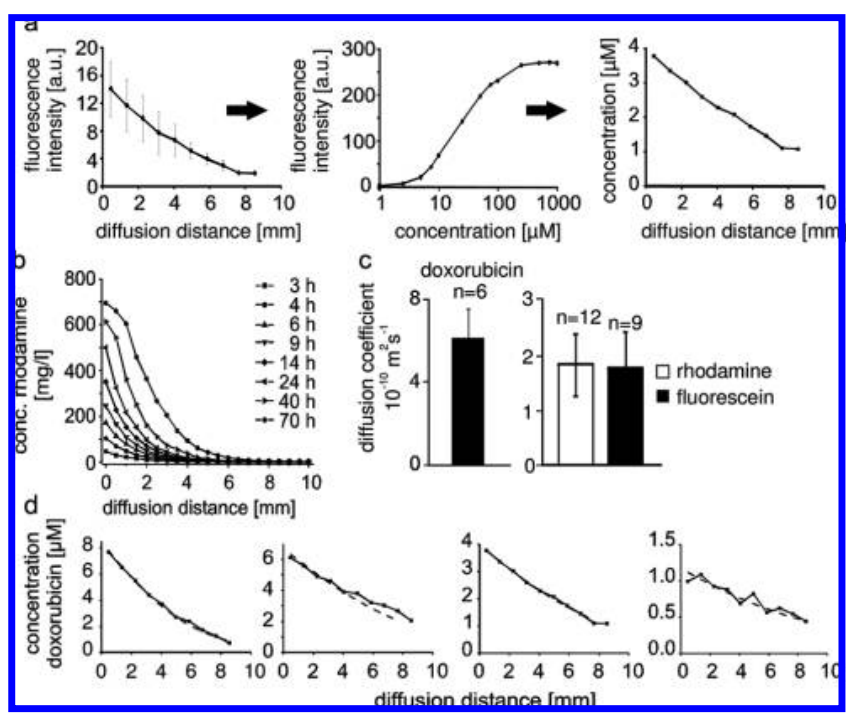

Figure 2. (a) Transformation of doxorubicin fluorescence intensities (left) to absolute concentrations (right) via a calibration curve (middle). Doxorubicin was loaded into the application slot at a concentration of $34.5 \mu \mathrm{M}$. (b) Time dependence of concentration profiles of rhodamine B. Diffusion proceeded in the dark at room temperature. At the indicated time points, the gradient was scanned. (c) Diffusion coefficients of doxorubicin (left) and rhodamine B and fluorescein (right) in agarose dissolved in medium including 10\% FCS. Diffusion coefficients were derived from curve fits with an error function describing diffusion from an infinite source. The error bars represent standard deviations from independent experiments. (d) Doxorubicin concentration gradients measured at different time points and with different starting concentrations compared with simulated concentration gradients assuming a diffusion coefficient of $6.14 \times 10^{-10} \mathrm{~m}^{2}$ $\mathrm{s}^{-1}$. The simulation model was based on diffusion from an infinite source. From left to right: $100 \mu \mathrm{M}$ starting concentration and $8 \mathrm{~h}$ diffusion time; $100 \mu \mathrm{M}$ starting concentration and $16 \mathrm{~h}$ diffusion time; $34.5 \mu \mathrm{M}$ starting concentration and $16 \mathrm{~h}$ diffusion time; $10 \mu \mathrm{M}$ starting concentration and $16 \mathrm{~h}$ diffusion time.

possible to provide reliable estimates for the time-dependent concentration gradient for any small molecule that would not interact with the diffusion matrix.

In order to validate the generation of concentration gradients by diffusion, we relied on fluorescent reference molecules. Next to fluorescein and rhodamine $\mathrm{B}$, the anticancer drug doxorubicin was used as a test compound (Figure 2). Upon excitation at 488 $\mathrm{nm}$, doxorubicin has two principal emission maxima at $\lambda_{1}=560$ and $\lambda_{2}=590 \mathrm{~nm} .{ }^{12}$ The three test compounds differed in their charge as well as water solubility, therefore representing a range of physicochemical characteristics specific for drugs. For all three compounds, calibration samples of known concentration were used to convert fluorescence intensities in the diffusion matrix into absolute concentrations (Supporting Information, Figure S-2).

As expected for entry by diffusion, the concentration gradients were time-dependent. For all three molecules, for the first $24 \mathrm{~h}$ the concentration profiles could be well described by an error function, assuming free diffusion from an infinite source. For diffusion times longer than $50 \mathrm{~h}$, the gradient could be better described by diffusion laws according to a limited source. Diffusion

(12) Karukstis, K. K.; Thompson, E. H.; Whiles, J. A.; Rosenfeld, R. J. Biophys. Chem. 1998, 73, 249-263. constants corresponded to those of free diffusion in water. ${ }^{13-16}$ Next to agarose, gelatin was successfully tested as a diffusion matrix (Figure S-3 in the Supporting Information). In principle, any material that enables a close contact of the device with the matrix and anchoring of the device via the burrs is suitable for generating concentration gradients.

Concerning the ratio of concentrations in the application slot and the concentration at the initial point in the assay area, for doxorubicin and fluorescein, we validated that over a concentration range of $10-500 \mu \mathrm{M}$ and a time of $4-24 \mathrm{~h}$, within an error margin of a factor of 2 , all dilution factors were in a range of a factor of 10. The concentration $c_{0}$ underneath the application slot that was required for the simulation of concentration gradients was a factor of 1.4 higher, corresponding to a dilution factor of 7 . The concentration range was time and temperature dependent. For rhodamine, diffusing at room temperature, after $24 \mathrm{~h}$, the concentration gradient reached from a 10-fold dilution next to the application slot to a 100 to 1000-fold dilution at the opposite site of the assay area. For doxorubicin diffusing at $37^{\circ} \mathrm{C}$, after $8 \mathrm{~h}$, the concentration range covered more than 1 order of magnitude. Furthermore, we confirmed that gradient formation remains unaffected by the presence of DMSO and DMF in concentrations of these solvents typically tolerated by cells. Pharmacologically active substances are frequently prepared as stock solutions in the presence of DMSO or DMF (Figure S-4 in the Supporting Information).

With this information for $D$ and $c_{0}$ at hand, it was possible to use the error function to predict concentrations for any position of the assay area and for any point in time. For nonfluorescent anticancer drugs (see below) for which the diffusion constant could not be determined experimentally, a diffusion coefficient of $6.14 \times 10^{-10} \mathrm{~m}^{2} / \mathrm{s}$, corresponding to the one determined for doxorubicin, was assumed, and $c_{0}$ was fixed to $1 / 7$ th of the concentration in the application slot.

Generation and Testing of Combinations of Cancer Drugs. Combination therapies are widely applied in the treatment of cancer. ${ }^{17,18}$ Therefore we explored if and how the diffusion device could be used for testing combinations of cancer drugs. In order to assess cytotoxicity, the MTT assay was adopted to be used in our diffusion device. The MTT test is based on the formation of a purple formazan salt precipitate by mitochondrial activity. When conducted in a standard microtiter plate format, this precipitate is dissolved. We reasoned that formation of the insoluble precipitate in a hydrogel matrix would preserve the local information on cell viability.

Cells were diluted into a solution of rat tail collagen in tissue culture medium that was pipetted into the assay area. After gelation, $200 \mu \mathrm{L}$ of medium was pipetted onto the collagen gel in order to prevent dehydration and provide nutrients to the cells. After an incubation of $16 \mathrm{~h}$, the medium was changed against a medium containing MTT. Formation of the purple precipitate was imaged by digital microscopy using a stereomicroscope followed by densitometry image analysis (Figure 3 ).

(13) Hansen, R. L.; Zhu, X. R.; Harris, J. M. Anal. Chem. 1998, 70, 1281-1287.

(14) Labille, J.; Fatin-Rouge, N.; Buffle, J. Langmuir 2007, 23, 2083-2090.

(15) Pluen, A.; Netti, P. A.; Jain, R. K.; Berk, D. A. Biophys. I. 1999, 77, 542552.

(16) Stewart, P. S. Antimicrob. Agents Chemother. 1996, 40, 2517-2522.

(17) Dancey, J. E.; Chen, H. X. Nat. Rev. Drug Discov. 2006, 5, 649-659.

(18) Kimby, E.; Nygren, P.; Glimelius, B. Acta Oncol. 2001, 40, 231-252. 


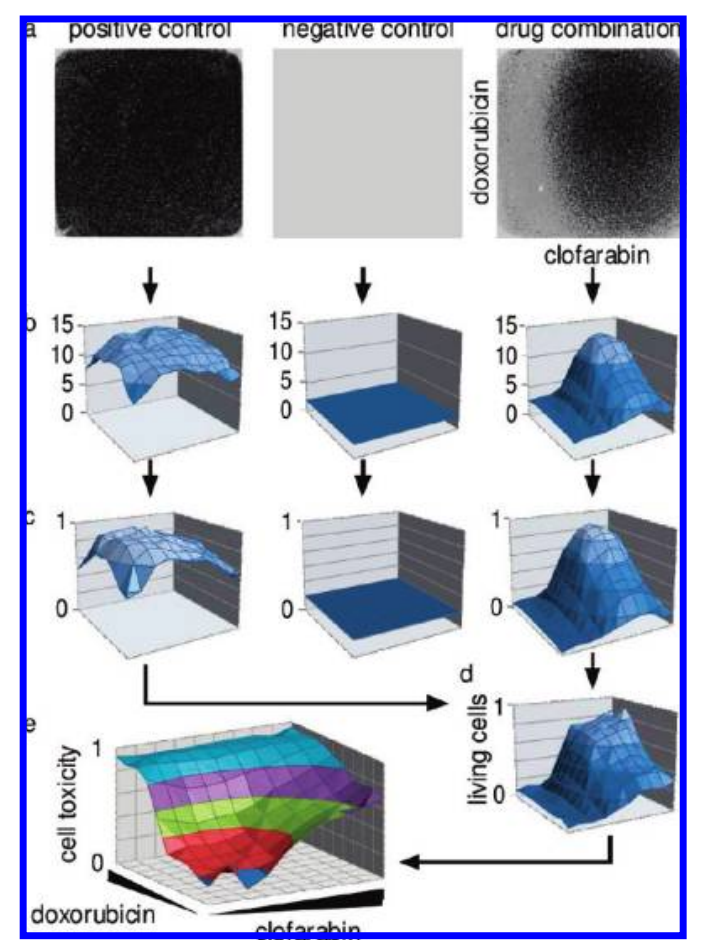

Figure 3. MTT-test in a diffusion device. Transmission images of the assay area (a) are converted into a matrix of gray values using a $10 \times 10$ point grid (b). (c) The response profile for the drug combination is corrected for background and expressed relative to a positive and a negative control using an image recorded for a matrix not containing any cells (negative control) and an image recorded from a device in which cells were not exposed to any drug (average gray scale value of the positive control). (d) The positive control is also used for correction of field heterogeneities. (e) Cytotoxicity is expressed as the inverse of cell viability. Here, a data set for MOLT-4 cells exposed to doxorubicin and clofarabin is shown.

In a first series of experiments, MOLT-4 acute lymphoblastic leukemia cells were used to identify an appropriate cell density and MTT concentration. Optimal results as judged by the required incubation time with the MTT reagent and the intensity of the staining were obtained using an MTT concentration of $1 \mathrm{mg} / \mathrm{mL}$ and a cell density of $7.5 \times 10^{6}$ cells $/ \mathrm{mL}$, which corresponds to $7.5 \times 10^{5}$ cells per device. This cell density is higher than the $5 \times 10^{5}$ cells/mL typically employed in MTT assays in the microtiter plate format. The read-out of the formazan absorption is subject to the Lambert-Beer law. In the central assay area, the thickness of the cell-containing gel is only $1 \mathrm{~mm}$ as compared to about $3 \mathrm{~mm}$ of solution in a well of the microtiter plate, explaining at least part of the difference in required cell density. Nevertheless, for a total of 100 data points, the number of cells used in the diffusion device $\left(7.5 \times 10^{5}\right.$ cells $)$ is only about a tenth of the amount needed for a standard microtiter plate $\left(5 \times 10^{6}\right.$ cells), therefore constituting a significant reduction in the required cell number.

Next, dose-response profiles were generated for doxorubicin, clofarabin, and etoposide. All three drugs are used in the clinic in the treatment of lymphoid and myeloid leukemias. ${ }^{18-21}$ At

(19) Gidwani, P.; Ramesh, K. H.; Liu, Y.; Kolb, E. A. Chemotherapy 2008, 54, 120-124.

(20) Muller, I.; Niethammer, D.; Bruchelt, G. Int.J. Mol. Med. 1998, 1, 491494.

(21) Sinkule, J. A. Pharmacotheraby 1984, 4, 61-73. concentrations of $34.5,10$, and $100 \mu \mathrm{M}$ in the application slot, respectively, after $16 \mathrm{~h}$ for all three compounds, clear activity profiles were obtained within the assay area (Figures 3 and 4). The concentrations of drugs in the application slots were based on educated guesses taking into account available information on bioactive concentrations and the dilution factor between the application slot and assay area. We were interested to learn to which degree the dose-response functions of drugs in the diffusion device compared to those with a standard microtiter plate assay. Cells are exposed to a time-dependent concentration gradient, and the induction of cell death may take several hours. As a consequence, higher concentrations of drug toward the end of the incubation period should contribute less to the biological response than those at the beginning. For this reason, we decided to use a time-weighted average concentration profile to provide an estimate for the effective concentration to which cells were exposed (Supporting Information, Figure S-5).

The diffusion device yielded dose-response profiles that compared well to those obtained in the standard 96-well microtiter plate format (Figure 4). From these dose-response profiles, the $\mathrm{EC}_{50}$ values for half maximal cell death for the single drugs and the drug combinations were derived. The $\mathrm{EC}_{50}$ values differed from the multiwell method to the diffusion device by a factor of 2 for clofarabin, 6 for etoposide, and 14 for doxorubicin. Nevertheless, for etoposide and doxorubicin and all combinations, the relative $\mathrm{EC}_{50}$ values had the same tendency for both assays. We identified the higher cell density in the diffusion device and to a minor degree the embedding in the collagen matrix as sources for these differences (Figure $\mathrm{S}-6$ in the Supporting Information). In the microtiter plate, a cell density of $1 \times 10^{6} / \mathrm{mL}$ had been employed in comparison to a cell density of $7.5 \times 10^{6} / \mathrm{mL}$ for the diffusion device. For doxorubicin, this difference in cell density alone accounted for a factor of 10 .

Ultimately, given the dependence of $\mathrm{EC}_{50}$ values on assay conditions, for the testing of drug combinations it is very important to learn whether their activity is additive, synergistic, or inhibitory. For this purpose we employed the combination index method (Figure 4c,d; Figure S-7 in the Supporting Information) ${ }^{22}$ The same profiles for the combination indices were obtained for the microtiter plate experiment and with the diffusion device. This result clearly indicates that even though the absolute concentration at which an effect is observed may vary, with respect to the activity of a drug combination, the response profiles led to the same conclusions

Next to the testing of drug combinations, the ability to rapidly derive dose-response relations of different cell types to certain drug combinations is a primary need in cancer research and oncology. Hence, three lymphoid leukemia (Jurkat, MOLT-4, and CEM) and three myeloid leukemia (K562, KG1A, and PLB598) cell lines were tested in parallel (Figure 5). K562 and Jurkat cells were insensitive to the applied drug combinations. The CEM cells showed some response to clofarabin and etoposide. PLB 598, MOLT-4, and KG1a cells were metabolically less active and showed different response profiles to the individual drug combinations. The differences in response profiles for KG1a and MOLT-4 cells were confirmed by analysis of the combination indices

(22) Chou, T. C. Pharmacol. Rev. 2006, 58, 621-681. 


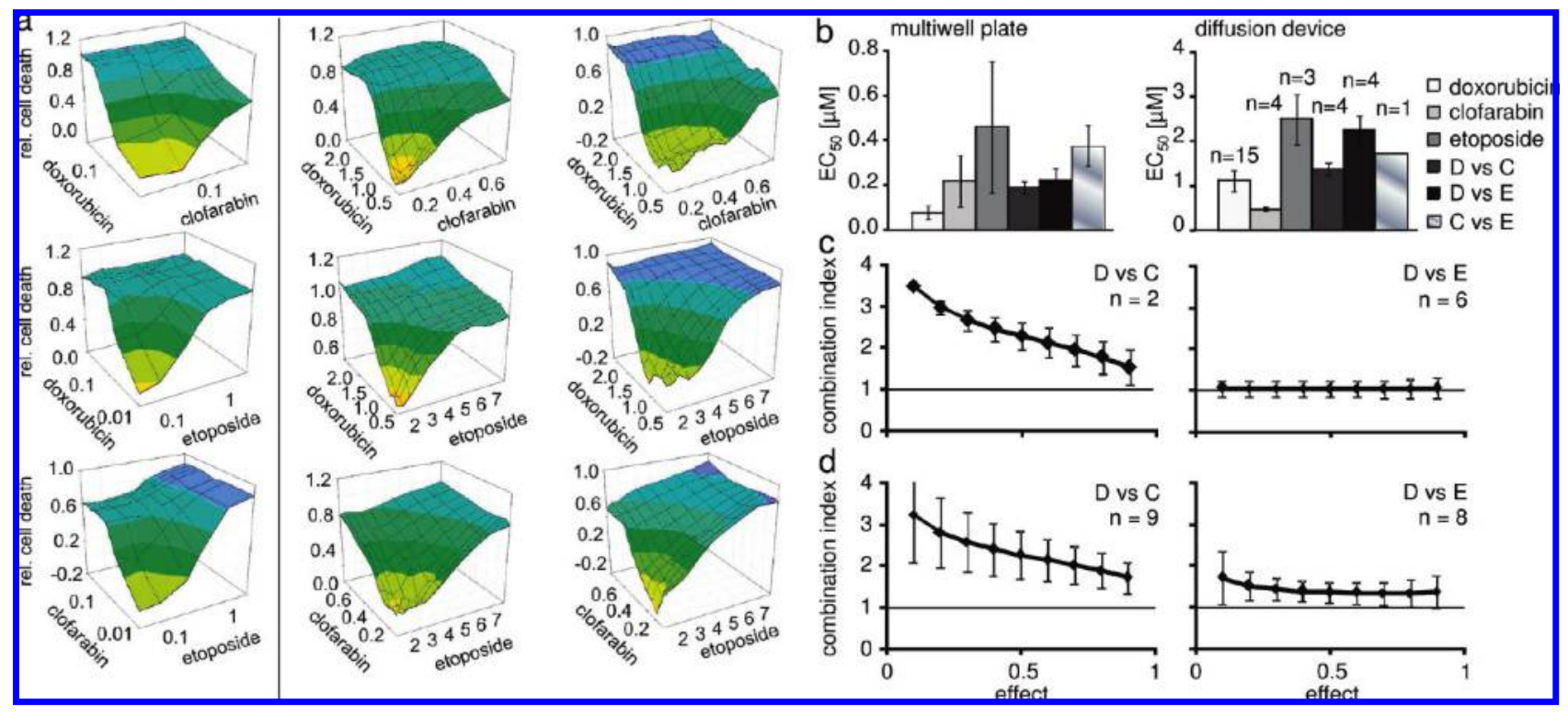

Figure 4. Comparison of the diffusion device with a standard microtiter plate experiment. Cytotoxicity assays were carried out with MOLT-4 lymphoid leukemia cells. The vertical axis indicates the response to the drug normalized to 1 for total cell killing. In the left column, results for the standard multiwell method are shown. The two columns on the right side are results from two independent experiments with the diffusion device. Drug concentrations for the diffusion device correspond to time-weighted averages (Figure S-4 in the Supporting Information). (b) EC $\mathrm{C}_{50}$ values for the drugs alone and in combination, determined with the diffusion device and in the microtiter plate format. For the diffusion device, the individual $\mathrm{EC}_{50}$ values were derived from the dose-response profile most distant to the other application slot. $\mathrm{EC}_{50}$ values for the combinations were determined along the diagonal. The determination of an $\mathrm{EC}_{50}$ value for clofarabin was complicated by the fact that also at the highest concentration of drug no full inhibition was achieved. (c, d) Classification of drug activity based on the combination index (Cl) over effect grade by Chou (Supporting Information). ${ }^{22}$ (c) Combinations from doxorubicin against clofarabin and etoposide tested against MOLT-4 cells in microtiter plates. (d) The same combinations and cell line in the diffusion device. Cl values $>1$ indicate antagonism, equal to 1 additive action, and $<1$ synergy. The error bars are standard deviations from independent experiments. Doxorubicin and etoposide both inhibit topoisomerase 2. Therefore, a mutually exclusive model was assumed. For clofarabin and the other two drugs, acting by different mechanisms of action, a mutually nonexclusive model was used.

(Figure S-8 in the Supporting Information). It should be noted that obtaining a comparable amount of information with a standard microtiter plate-based assay would have required 4320 pipetting steps (180 steps per drug combination for 10 by 10 concentrations) while with the diffusion device, the same amount of data points can be obtained with only 24 pipetting steps.

The ability to acquire detailed dose-response profiles with minimum handling steps and cells renders the diffusion device a highly attractive tool for testing the drug responsiveness of primary patient isolates, both in preclinical drug development as well as in personalized medicine. However, in the case of leukemia, a major challenge is the poor viability of primary leukemic cells ex vivo. ${ }^{23-25}$ In order to evaluate the diffusion device for the testing of primary cells, leukemic cells obtained from a pediatric patient diagnosed with T-lineage acute lymphoblastic leukemia (T-ALL) were tested in the diffusion device (Figure 5). In comparison to the leukemia cell lines, a significantly higher number of cells, $3.0 \times 10^{6}$ in comparison to $7.5 \times 10^{5}$ per device, was needed to enable efficient conversion of the MTT substrate. Nevertheless, clear dose-response profiles that depended on drug concentration in the application slot were obtained after an incubation time of $16 \mathrm{~h}$. The mean of $\mathrm{EC}_{50}$

(23) Backman, E.; Bergh, A. C.; Lagerdahl, I.; Rydberg, B.; Sundstrom, C.; Tobin, G.; Rosenquist, R.; Linderholm, M.; Rosen, A. Haematologica 2007, 92, 1495-1504.

(24) Ryningen, A.; Ersvaer, E.; Oyan, A. M.; Kalland, K. H.; Vintermyr, O. K.; Gjertsen, B. T.; Bruserud, O. Leuk. Res. 2006, 30, 1531-1540.

(25) Savitskiy, V. P.; Shman, T. V.; Potapnev, M. P. Cytometry, Part B 2003, $56,16-22$. values from a total of four experiments performed with different concentrations in the application slot was $1.8 \pm 0.2 \mu \mathrm{M}$. In the clinic, plasma concentrations of $1-5 \mu \mathrm{M}$ are reached. ${ }^{26,27}$ Therefore, the $\mathrm{EC}_{50}$ values determined by our approach compare very favorably with these clinical concentrations.

Implementation of an Annexin V-Based Assay of Cell Death. The MTT assay is a general cell viability assay that does not allow conclusions to be drawn on the particular mechanism of action of a compound. In the testing of anticancer agents, it is of great interest to learn whether a compound affects metabolic activity or cell growth or induces cell death either by necrosis or apoptosis. Phosphatidylserine exposure to the outer leaflet of the plasma membrane is a characteristic of early apoptosis and necrosis that can be detected by binding of fluorescently labeled annexin $\mathrm{V}^{28}$ Doxorubicin is known to induce apoptotic cell death. ${ }^{27}$ Fluorescently labeled annexin $\mathrm{V}$ was dissolved in the collagen matrix throughout the experiment. Phosphatidylserine exposure led to a local sequestration of annexin $\mathrm{V}$ that enabled a clear discrimination of cells from their environment (Figure 6a). Confocal detection was employed in order to suppress blurr from out-of-focus light. In order to correct dose-response gradients for local heterogeneities in cell density, cells were counterstained

(26) Hempel, G.; Flege, S.; Wurthwein, G.; Boos, J. Cancer Chemother. Pharmacol. 2002, 49, 133-141.

(27) Muller, I.; Jenner, A.; Bruchelt, G.; Niethammer, D.; Halliwell, B. Biochem. Biophys. Res. Commun. 1997, 230, 254-257.

(28) Vermes, I.; Haanen, C.; Steffens-Nakken, H.; Reutelingsperger, C. I. Immunol. Methods 1995, 184, 39-51. 


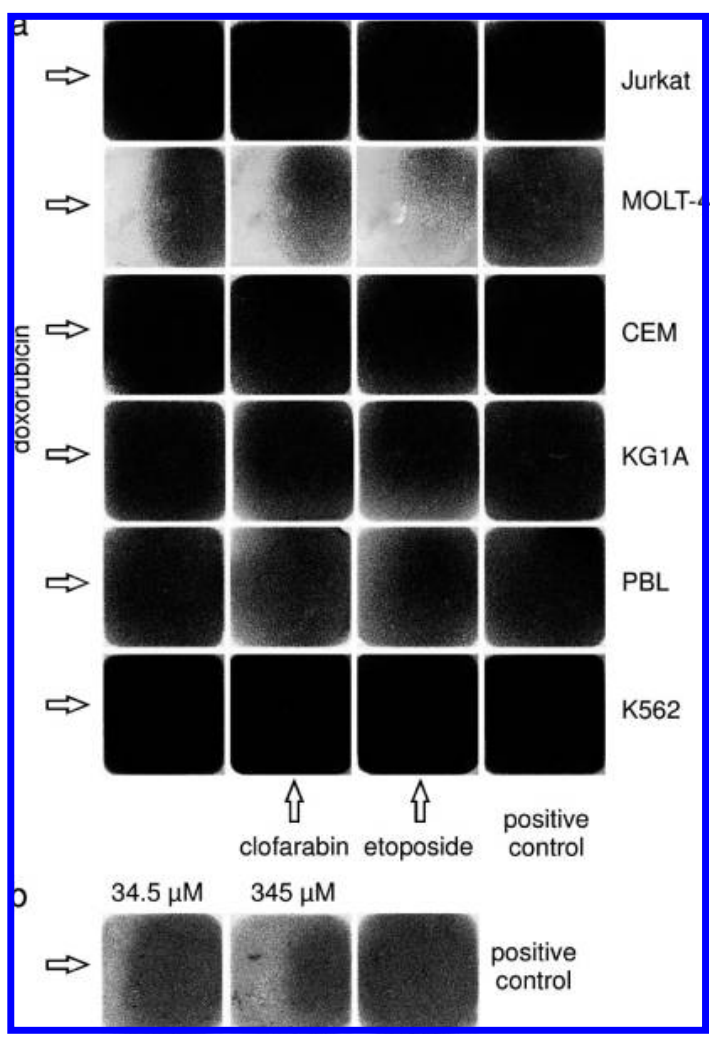

Figure 5. Comparison of dose-response profiles. (a) Testing of dose-response profiles for six leukemia cell lines in parallel. The arrows indicate the direction of diffusion for the different drugs. All cells were applied at a density of $7.5 \times 10^{6}$ cells $/ \mathrm{mL}$. Assays were carried out for $16 \mathrm{~h}$. The right column shows positive controls not treated with any drug. (b) Testing of primary ALL-patient cells with two different starting concentrations of doxorubicin. For the leukemia cell lines, $7.5 \times 10^{5}$ cells were used per device and for the ALL cells, $3.0 \times 10^{6}$ cells.

with the celltracer carboxyfluoroescein-succinimidylester. Doxorubicin induced clear gradients of annexin V-positive cells (Figure $6 c, d)$. Confocal microscopy at higher magnification confirmed that the gradients of annexin $\mathrm{V}$ fluorescence resulted from membranebound fluorescence (Figure 6b). Furthermore, these results demonstrate the ability to address cellular characteristics in the diffusion device down to the subcellular level.

\section{DISCUSSION}

We have developed a novel diffusion-based method for highresolution dose-response profiling of drug interactions. This method, which involves a simple diffusion device, yields a tremendous reduction of processing steps for the determination of dose-response profiles of cells to drug combinations. Diffusion is a powerful means for the generation of concentration gradients that is governed by well-understood physical laws. These physical laws also define the boundary conditions for assays with the diffusion device, especially the time required for formation of a concentration gradient. For small molecule cancer drugs, the few hours needed to establish a concentration gradient were fully compatible with cytotoxicity assays in which cells and drugs were added at the same time.

With the MTT test, an established assay for the quantification of cell viability could be transferred to the format of the diffusion device that yielded results that led to the same conclusions on

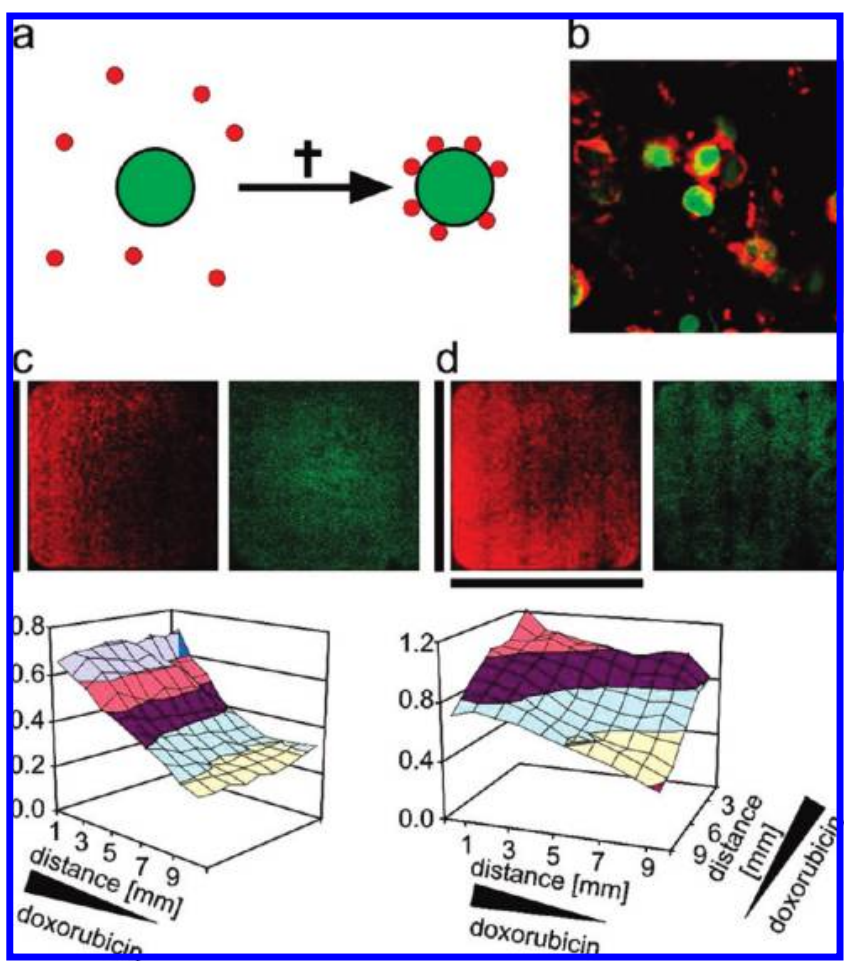

Figure 6. Detection of doxorubicin-induced cell death by annexin $\mathrm{V}$ staining of phosphatidylserine exposure. (a) Assay principle: Upon phosphatidylserine exposure, AlexaFluor 647-labeled annexin V, (red) which is homogeneously distributed in the collagen matrix, binds to cells, leading to a local enrichment of fluorescence. Cells are counterstained by incubation with carboxyfluorescein succinimidylester (green). The counterstaining enables a correction of apoptosis gradients for heterogeneities in cell density. (b) Annexin V-positive cells at higher magnification. The collagen film was lifted off the diffusion matrix, placed on a microscopy slide, and imaged by confocal microscopy. (c) One and (d) two-dimenstional gradients of doxorubicin, employed at a starting concentration of $345 \mu \mathrm{M}$ in the application slot and recorded after $16 \mathrm{~h}$. Top, Fluorescence images of annexin V AlexaFluor 647(red) and the CFSE stain (green); bottom, gradients of annexin $\mathrm{V}$-positive cells calculated by dividing fluorescence in the annexin $\mathrm{V}$ channel through fluorescence in the CFSE channel. A total of $4 \times 10^{5} \mathrm{MOLT}-4$ cells were used in the devices. The positions of the application slots are indicated.

drug interactions as assays conducted in the microtiter plate format.

The validation of this assay in comparison to a standard microtiter plate-based assay faced two challenges: First, the determination of concentrations and second the nonstationary behavior of the concentration gradients. Using intrinsic fluorescence for detection, for three different small molecules including the cancer drug doxorubicin, we validated that concentration gradients were formed by free diffusion. While it cannot be excluded that certain molecule interactions with the diffusion matrix may inhibit diffusion, in most cases the concentration gradients may be calculated from the factor 10 offset between the concentrations in the application slot and the diffusion matrix, the diffusion constant, and the diffusion time.

In spite of the nonstationary behavior of the gradients, with a total assay time of $16 \mathrm{~h}$ for the MTT-test, the cells showed a graded response over the whole assay area. Assuming a time-weighted average concentration for each location within the device, $\mathrm{EC}_{50}$ values determined by the diffusion device in comparison to the 
microtiter plate-based assay differed by a factor of 2-14, depending on the drug used. Preformation of the gradient over $4 \mathrm{~h}$ followed by incubation of the cells on the matrix over $12 \mathrm{~h}$ had little effect on the difference between the microtiter platebased assay and the results obtained with the diffusion device. Instead, we determined experimentally that the cell density explained these differences for the major part and the embedding in the collagen for the minor part. A negative correlation of drug sensitivity and cell density in leukemic cancers has been observed before. ${ }^{29}$ Therefore, the time-weighted average concentrations provide a reasonable estimate of the drug dose to which the cells are exposed.

In the comparison of drug sensitivities, some cell lines proved to be resistant toward the tested drugs (Figure 5). To this point, we did not address whether cell death occurred at later time points. Our simulations had shown that after $24 \mathrm{~h}$, the gradients slowly faded away. It will therefore be interesting to test whether after longer incubation times, graded responses reflecting the time-dependent dose-response profile may still be observed. In the context of these experiments it will also be interesting to explore to which degree the diffusion device may be useful for compounds that act via different mechanisms-of-action such as kinase and proteasome inhibitors.

Next to the MTT-assay we present the adaptation of an annexin V-based assay of cell death to the format of the diffusion device. Because of the incorporation of annexin V into the collagen matrix, the local sequestration of this protein to cells with phosphatidylserine exposed on the outer leaflet of the plasma membrane may be followed throughout the experiment and does not require further staining steps. This experiment also demonstrated the compatibility of the assay format with the microscopy of single cells. Ultimately, a thorough discrimination of apoptosis versus necrosis-induced phosphatidylserine exposure requires a further stain to probe for maintenance of plasma membrane integrity during the early steps of apoptosis. ${ }^{28}$ Nevertheless, these results demonstrate that also fluorescence-based assays can be elegantly adapted to the format of the diffusion device.

A point that deserves further attention is in how far the time-concentration profile may lead to cellular responses that differ from those of a drug or drug combination in a clinical application. If in the diffusion device cells are present during formation of the gradient, then cells are initially exposed to low drug concentrations that may induce the expression of proteins that antagonize drug action. In a typical clinical application in cancer therapy, cells are exposed to a rapid increase to high drug concentrations which are then maintained or fall of more slowly. However, potential differences in cellular responses will be less pronounced or negligible if cells are added to the diffusion device after formation of the gradient.

The testing of dose-response profiles for thousands of drug combinations is a challenge that will be difficult to meet with even the most powerful pipetting robots. Here, the diffusion assay combines an old principle for the generation of concentration

(29) Masquelier, M.; Vitols, S. Biochem. Pharmacol. 2004, 67, 1639-1646. gradients with advanced technology for the detection of concentration gradients and assay read-outs.

Furthermore, the generation of up to 100 data points with only $7.5 \times 10^{5}$ cells in a total assay volume of $100 \mu \mathrm{L}$ represents a considerable miniaturization. Typically, assay miniaturization is hampered by the increasing surface to volume ratio of the assay cavities, and increased sample-to-sample variance due to larger relative errors in the processing of the assay and evaporation. As our assay is performed in a single well, the diffusion device circumvents problems commonly associated with miniaturization.

For the testing of primary leukemia cells, $3.0 \times 10^{6}$ cells were needed per device. Given the fact that typically $10^{8}$ to $10^{9}$ cells are obtained from a patient with a hematologic malignancy, large numbers of tests on drug-response profiles can be performed.

\section{CONCLUSIONS}

At a time, when because of the high costs of new chemical entities, there is increasing emphasis on combination therapies, the diffusion device addresses a major need of biomedical research, preclinical drug development, and the pharmaceutical industry. Especially, with the advent of numerous new therapy options in cancer therapy arising through the introduction of kinase inhibitors ${ }^{30}$ and other small molecule drugs such as proteasome inhibitors, ${ }^{31}$ there is a vast landscape in drug space that has to be explored for novel optimum drug combinations. ${ }^{32}$ Furthermore, in view of the small number of cells required for this assay in relation to the numbers of cells typically obtained in hematological malignancies, our device could provide a convenient assay for the rapid determination of drug interactions in individual patients as a first step toward personalized medicine in hematological cancers. ${ }^{3}$ It will be interesting to explore applications in other types of cancers with a more limited availability of cells.

\section{ACKNOWLEDGMENT}

A.G. and G.R. contributed equally to this work. We thank A. Treftz for the manufacturing of the diffusion devices. We gratefully acknowledge financial support from the Volkswagen Foundation ("Nachwuchsgruppen an Universitäten" Grant I/77 472) and the German Ministry of Education and Research (Grants FKZ 13N8646, 13N8647, and 13N9142).

\section{SUPPORTING INFORMATION AVAILABLE}

Additional information as noted in text. This material is available free of charge via the Internet at http://pubs.acs.org.

Received for review February 24, 2009. Accepted May 12, 2009.

\section{AC900415S}

(30) Krause, D. S.; Van Etten, R. A. N. Engl. I. Med. 2005, 353, 172-187.

(31) Nencioni, A.; Grunebach, F.; Patrone, F.; Ballestrero, A.; Brossart, P. Leukemia 2007, 21, 30-36.

(32) Ocio, E. M.; Mateos, M. V.; Maiso, P.; Pandiella, A.; San Miguel, J. F. Lancet Oncol. 2008, 9, 1157-1165. 